\title{
CELLULAR EXPRESSION OF RENAL, CARDIAC AND PULMONARY INDUCIBLE NITRIC OXIDE SYNTHASE IN DOUBLE-TRANSGENIC MICE EXPRESSING HUMAN RENIN AND ANGIOTENSINOGEN GENES
}

\author{
Zaher A Radi* and Yahya Murad \\ *Drug Safety Research and Development, Pfizer Global Research and Development, St Louis, Misouri and ${ }^{\dagger}$ University of \\ Michigan Medical School, Geriatrics Center and Institute of Gerontology, Ann Arbor, Michigan, USA
}

\section{SUMMARY}

1. Hypertensive mice expressing the human renin (REN) and angiotensinogen (AGT) genes are used as a model for human hypertension.

2. The aim of the present study was to investigate the cellular expression and distribution of inducible nitric oxide synthase (iNOS) using immunohistochemistry in lung, heart and kidney tissues from a model of human hypertension using male and female double-transgenic (h-Ang 204/1h-Ren6) mice and wild-type C57/BI6J mice as controls.

3. In the kidney, the pattern of iNOS expression in various renal microanatomical regions during hypertension was similar to that of age-matched controls, except in the medullary ascending limb (MAL). In hypertension, iNOS expression was downregulated in the MAL. No significant differences in iNOS expression were seen between control or hypertensive mice in various cardiac microanatomical locations. In the lungs of hypertensive mice, iNOS expression was upregulated in bronchial airway epithelium and bronchial and vascular smooth muscle cells, but downregulated in alveolar macrophages, alveolar septa and pulmonary vascular endothelial cells. Expression of iNOS was similar between male and female mice in the kidney, heart and lungs.

4. In conclusion, iNOS regulation in hypertension is complex and depends on the cell type in which it is expressed and the localization of the cell type in the cardiorenal and pulmonary systems.

Key words: angiotensin, hypertension, inducible nitric oxide synthase, renin.

\section{INTRODUCTION}

The renin (REN)-angiotensin-aldosterone system (RAAS) plays an important role in the control of cardiovascular and renal homeostasis by regulating blood pressure (BP) and fluid volume. ${ }^{1,2}$ Angiotensin (Ang) II is produced via an enzymatic cascade that begins with angiotensinogen (AGT) cleaving REN to form AngI, which is then

Correspondence: Zaher A Radi, Pfizer Global and Research Development, St Louis Laboratories, 700 Chesterfield Parkway West, Building BB371-2 (BB3N), St Louis, MO 63017, USA. Email: zaher.radi@ pfizer.com

Received 7 September 2008; revision 6 October 2008; accepted 24 October 2008

(C) 2009 The Authors

Journal compilation (C) 2009 Blackwell Publishing Asia Pty Ltd cleaved by angiotensin-converting enzyme (ACE) to form AngII. ${ }^{3}$ The genes for REN and AGT have been linked to, or associated with, hypertension in animal models and humans. ${ }^{2}$ Transgenic rodent models have been developed that overexpress both human REN and AGT, which leads to hypertension via chronic overproduction of AngII. Specific examples include the murine double-transgenic line (h-Ang 204/1h-Ren 9), which produces a mean arterial BP $40 \mathrm{mmHg}$ higher than wild-type mice (C57B1/6J) that lack the human genes. ${ }^{2}$ In addition, transgenic rats harbouring the mouse $R E N-2$ gene developed hypertension, cardiac hypertrophy and renal damage. ${ }^{4}$ Tsukuba hypertensive mice (THM), which express human REN and AGT genes, have also been shown to develop hypertension. ${ }^{5}$ There is increasing evidence suggesting that a RAAS may reside within several organs, including the kidney, lung, heart and in vascular smooth muscle cells (VSMC), where it is believed to act in a functionally independent paracrine/autocrine fashion. ${ }^{6}$ This hypothesis is further supported by the fact that all components of the RAAS in the heart, kidney and lung contain the ACE component. ${ }^{3,6}$ In addition, high concentrations of AngII have been demonstrated in the plasma, heart and kidney of THM. ${ }^{7.8}$

Inducible nitric oxide synthase (iNOS) is a calcium-independent inducible isoform of nitric oxide synthase (NOS) that can be induced by various cytokines. ${ }^{9}$ In the kidney, nitric oxide (NO) and iNOS may have various roles, including in hypertension. ${ }^{9}, 10$ Inhibition of NO synthesis has also been demonstrated to decrease plasma REN activity ${ }^{11}$ and long-term inhibition of NO leads to AngII-dependent hypertension. ${ }^{12}$ Therefore, NO appears to have a role in RAASmediated hypertension.

There are currently no published reports on the cellular expression and microanatomical distribution of iNOS in hypertensive doubletransgenic mice expressing human REN and AGT genes. Therefore, using immunohistochemistry, we investigated the microanatomical location and cellular expression of iNOS in kidney, lung and heart tissues obtained from REN-AGT-transgenic mice and age-matched controls. The present study is the first to report on the pulmonary and cardiorenal microanatomical cellular expression and distribution of iNOS and possible mechanistic pathophysiological insights in this model of human hypertension.

\section{METHODS}

\section{Animals}

The present study used 15-20-week-old male $(n=15)$ and female $(n=15)$ double-transgenic mice (h-Ang 204/1h-Ren 9). The mice were derived from 
a founder colony of five male mice expressing human AGT (h-Ang 204/1) and six females expressing human REN (h-Ren 6), obtained from Dr Curt Sigmund at the University of Iowa School of Medicine (Iowa City, IA, USA). At Charles River Laboratories (Wilmington, MA, USA), female mice that expressed human REN were bred with AGT-expressing male mice to produce the double-transgenic line. The transgenic line was developed on a C57/BI6J background. Details of the generation and phenotype of the double transgenic mouse are described elsewhere. ${ }^{13,14}$ Age-matched male $(n=10)$ and female $(n=10)$ C57/BI6J mice were used as controls. All procedures were in compliance with the Pfizer Ann Arbor Laboratories Animal Care and Use Committee.

\section{Haemodynamic parameters}

The haemodynamic changes in this model have been well characterized previously.$^{15}$ Briefly, transgenic mice were prepared with in-dwelling aortic catheters and radiotransmitters (Data Sciences International, St Paul, MN, USA). The catheters were placed in the left carotid artery and advanced to the aortic arch. The radiotransmitters allowed continuous measurement of the aortic $\mathrm{BP}$ waveform, from which mean arterial pressure (MAP) and heart rate (HR) were derived using Ponemah software (Gould Instruments, Valley View, OH, USA).

\section{Study tissue samples}

Lung, kidney and heart tissues were obtained from mice at the time of necropsy. Tissues were fixed in $10 \%$ neutral buffered formalin for $24 \mathrm{~h}$ and embedded in paraffin wax. Sections $(3 \mu \mathrm{m})$ were then cut and stained immunohistochemically with antibodies against iNOS.

\section{Immunohistochemistry for iNOS}

To analyse the expression of iNOS, $3 \mu \mathrm{m}$ sections were cut from formalinfixed, paraffin-embedded blocks, mounted on positively charged glass slides, dried and then loaded on the automated immunostainer at room temperature using a Ventana Immunostainer System (Ventana Medical Systems, Tucson, AZ, USA). Slides were deparaffinized and then rehydrated. Sections were incubated for $30 \mathrm{~min}$ with serum-free DakoCytomation protein blocker (Dako Corporation, Dako, CA, USA) and then rinsed and incubated for 4 min with avidin-biotin blocking solution (Ventana Medical Systems). Antigen retrieval was completed with a Ventana specialty solution $(\mathrm{pH}=8$; Ventana Medical Systems).

Automation included exposure to $100 \mu \mathrm{L}$ primary anti-iNOS antibody (Cayman Chemical, Ann Arbor, MI, USA) diluted $1: 100$ with reagent diluent (Ventana Medical Systems) at room temperature for $60 \mathrm{~min}$, followed by the addition of $100 \mu \mathrm{L}$ of the appropriate anti-rabbit biotinylated IgG linking solution (1:200 dilution; Vector Laboratories) to each section for $60 \mathrm{~min}$ at room temperature. Sections were rinsed with buffer and allowed to react with $100 \mu \mathrm{L}$ diaminobenzidine (DAB) substrate solution (DAB Detection Kit; Ventana Medical Systems) for $8 \mathrm{~min}$, followed by counterstaining with haematoxylin and then bluing reagent for $4 \mathrm{~min}$ each, removed from the autostainer, washed in warm water, dehydrated through graded alcohol, cleared in xylene and cover slipped. Control reactions included: (i) sections incubated with the omission of primary antibody, but otherwise processed as described above; and (ii) sections incubated with normal rabbit serum instead of the primary antibody and the processed as described above.

Expression of iNOS was evaluated semiquantitatively. Staining (percentage of immunostained cells) was scored as follows: -, no staining; + , minimal staining; ++ , mild staining $(<30 \%$ of the cell population stained); +++ , moderate staining $(<60 \%$ of the cell population stained); and ++++ , strong staining ( $>60 \%$ of cells stained).

\section{RESULTS}

Pulmonary and cardiorenal cellular expression and distribution of iNOS are summarized in Tables 1 and 2. Staining intensity ranged from negative (-) to strong (++++).
Table 1 Renal immunohistochemical expression of inducible nitric oxide synthase

\begin{tabular}{lll}
\hline Cellular location & Control & Hypertension \\
\hline Macula densa & ++ & ++ \\
Proximal convoluted tubules & +++ & ++ \\
Distal convoluted tubules & +++ & ++ \\
VSMC & - & - \\
Vascular EC & + & - \\
Cortical IC & ++ & + \\
Medullary IC & ++ & + \\
Glomeruli (podocytes) & - & - \\
Glomerular (visceral) epithelium & + & - \\
Capsular (parietal) epithelium & + & - \\
Cortical collecting ducts & +++ & +++ \\
Medullary collecting ducts & +++ & +++ \\
MAL & +++ & + \\
\hline
\end{tabular}

-, no staining; +, minimal staining; ++, mild staining; +++, moderate staining; VSMC, vascular smooth muscle cells; EC, endothelial cells; IC, interstitial cells; MAL, medullary ascending limb.

Table 2 Pulmonary and cardiac immunohistochemical expression of inducible nitric oxide synthase

\begin{tabular}{lll}
\hline Cellular location & Control & Hypertension \\
\hline Alveolar macrophages & +++ & + \\
Alveolar septa & +++ & + \\
Bronchial epithelium & - & ++ \\
Bronchial SMC & + & ++++ \\
Bronchiolar epithelium & - & - \\
Pulmonary vascular EC & +++ & - \\
Pulmonary VSMC & ++ & ++++ \\
Cardiac myocytes & +++ & ++++ \\
Cardiac VSMC & + & - \\
Cardiac vascular EC & - & - \\
\hline
\end{tabular}

-, no staining; + , minimal staining; ++ , mild staining; +++, moderate staining; ++++, strong staining; VSMC, vascular smooth muscle cells; EC, endothelial cells; SMC, smooth muscle cells.

In the kidney of hypertensive mice, moderate (+++) diffuse cytoplasmic iNOS expression occurred in the distal convoluted tubules, cortical collecting ducts and medullary collecting ducts, whereas mild (++) iNOS expression was observed in the macula densa and proximal convoluted tubules (Fig. 1; Table 1). This pattern of iNOS expression was similar to that of age-matched control mice. Minimal (+) diffuse iNOS cytoplasmic staining was present in cortical and medullary interstitial cells (IC) and the medullary ascending limb (MAL) in hypertensive mice. Conversely, in control mice, mild (++) diffuse iNOS cytoplasmic expression was observed in cortical and medullary IC and moderate (+++) staining was seen in the MAL. In hypertensive mice, iNOS expression was lacking in the glomerular visceral epithelium, capsular parietal epithelium, glomerular podocytes, vascular endothelial cells (EC) and VSMC. This expression pattern was similar to that of controls, with the exception of minimal iNOS expression in vascular EC, glomerular visceral epithelium and capsular parietal epithelium.

In hypertensive mice, pulmonary expression of iNOS included strong diffuse staining in bronchial smooth muscle cells (SMC) and 


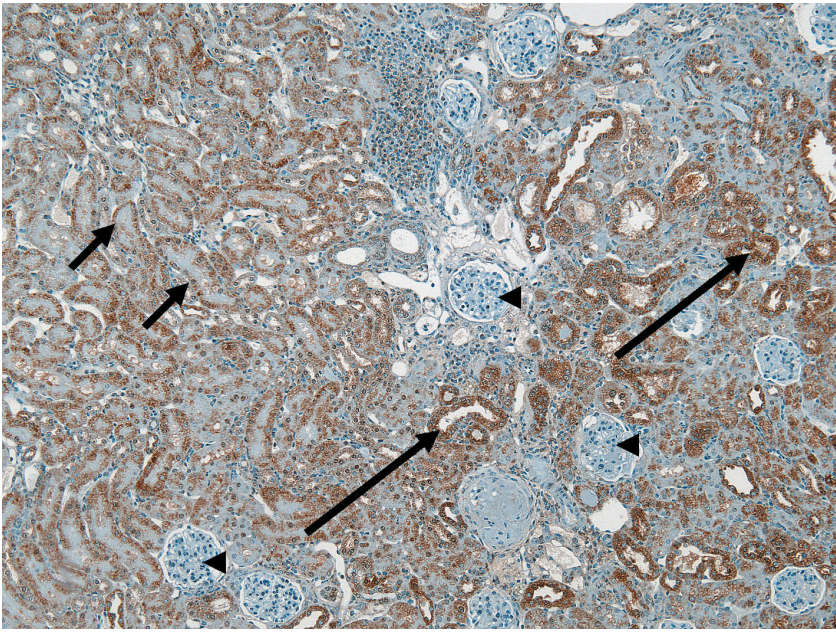

Fig. 1 Inducible nitric oxide synthase expression in the kidney in hypertension. There is moderate expression in the distal convoluted tubules and cortical collecting ducts (long arrows). Note the mild expression in the proximal convoluted tubules (short arrows). Immunohistochemical stain (original magnification $\times 10$ ).

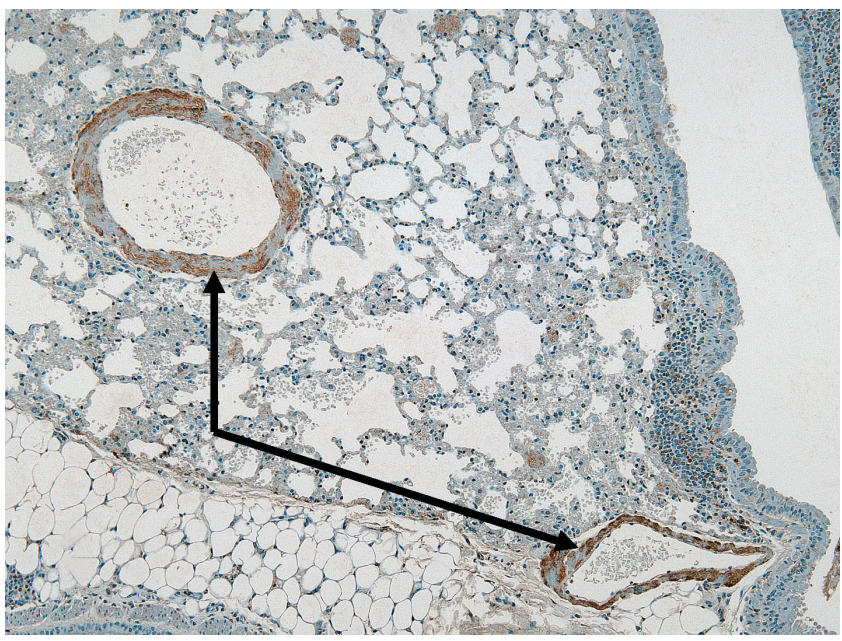

Fig. 2 Inducible nitric oxide synthase expression in the lung in hypertension. Strong expression is seen in the bronchial and vascular smooth muscle cells (long arrows). Immunohistochemical stain (original magnification $\times 10$ ).

VSMC (Fig. 2; Table 2), whereas minimal (bronchial SMC) and mild (VSMC) expression was present in lungs from control mice. Mild staining was present in the bronchial epithelium and minimal staining was present in the alveolar macrophages and septa. In control mice, moderate staining was observed in alveolar macrophages and septa, and no expression was present in the bronchial epithelium. There was no iNOS expression in the bronchiolar epithelium of lungs from either normal or hypertensive mice. In addition, there was no iNOS expression in the vascular EC in lungs from hypertensive mice, whereas in control mice moderate iNOS expression was found in the vascular EC.

Cardiac myocytes from both hypertensive and control mice had moderate to strong iNOS expression, whereas cardiac vascular SMC and EC from hypertensive mice were negative for iNOS (Fig. 3; Table 2). In control mice, minimal iNOS expression was present in

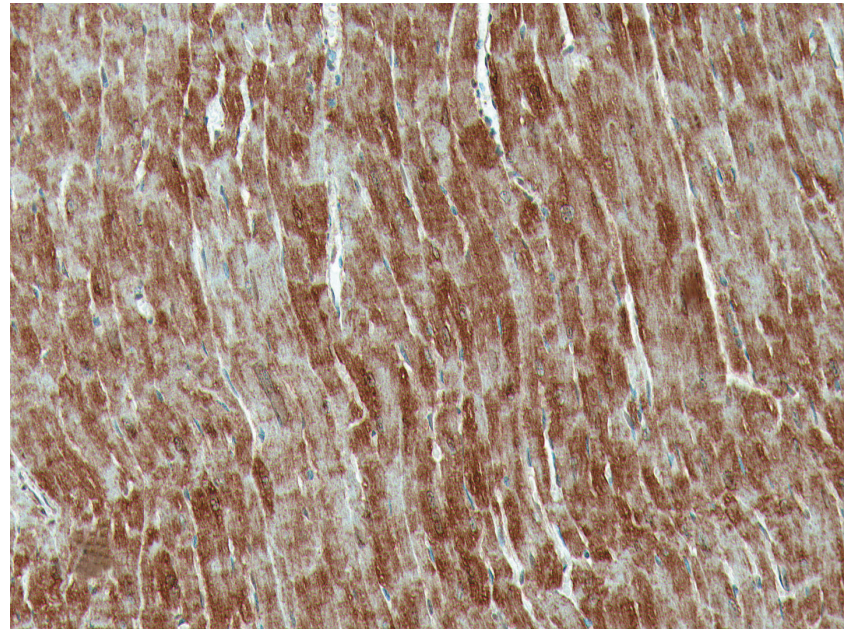

Fig. 3 Inducible nitric oxide synthase expression in the heart in hypertension. Strong expression in cardiac myocytes is observed. Immunohistochemical stain (original magnification $\times 10$ ).

cardiac VSMC and there was no iNOS expression in cardiac vascular EC. All these expression patterns for iNOS were similar between male and female mice.

\section{DISCUSSION}

Within the kidney, NO contributes to the regulation of glomerular haemodynamics and blood pressure control. ${ }^{10,16,17}$ In fact, the gene locus coding for iNOS has been shown to cosegregate with blood pressure in the Dahl salt-sensitive rat $^{18}$ and blood pressure is increased in iNOS-deficient mice compared with that in wild-type controls. ${ }^{19}$ In addition, continuous infusion for 6 days of aminoguanidine, a selective iNOS inhibitor, increased MAP in rats. ${ }^{10}$ Although iNOS is only induced by pro-inflammatory factors in most tissues, in situ hybridization and reverse transcription-polymerase chain reaction experiments have shown that iNOS is expressed constitutively in the renal medulla and proximal tubules and to a high degree in various regions of the normal rodent kidney, where iNOS participates in the regulation of renal function and arterial pressure. ${ }^{20,21} \mathrm{In}$ the present study, the overall pattern of iNOS expression in the kidney was similar to that of age-matched control mice, with the exception of downregulation of iNOS expression in the MAL in hypertensive mice. The high degree of iNOS expression in normal mice in the MAL region of the kidney in the present study is consistent with previous results of iNOS localization primarily in the MAL region of the normal rodent kidney. ${ }^{22}$ Mattson and Higgins have demonstrated, using protein blotting, that greater amounts of iNOS immunoreactive protein exist in rat renal medulla tissue homogenates. ${ }^{23}$ Nitric oxide derived from the renal medulla has a known role in the control of vasa recta blood flow, as well as sodium and water excretion. ${ }^{24,25}$ It has been found that inhibition of NO synthesis predisposes both animals and humans with pre-existing renal damage to severe necrosis of the MAL. ${ }^{24,25}$ The mechanism of hypertension after iNOS inhibition with aminoguanidine in rats is thought to be related to decreased renal sodium and water excretion, which leads to extracellular volume expansion. $^{10}$

The downregulation of iNOS expression in the MAL region in hypertensive mice is consistent with the role that MAL may play in 
sodium haemostasis and hypertension. ${ }^{26}$ In addition, It has been suggested that renal medullary oxidative stress may affect blood pressure and sodium balance via changes in NO, which inhibits sodium chloride reabsorption by the MAL and collecting duct. ${ }^{26}$ Hypertension may also result directly from pathological alterations to transporters, channels or enzymes that affect medullary NO. ${ }^{26}$ It has been suggested that the mechanism of some antihypertensive drugs, such as captopril, may involve modulation of NO activity in specific microanatomical regions of the kidney. ${ }^{27}$ For example, NOS activity of the renal cortex was significantly increased in spontaneously hypertensive rats after treatment with the antihypertensive drug captopril. ${ }^{27}$

The results of the present study regarding iNOS expression in other renal microanatomical locations are consistent with those of studies, which demonstrated that iNOS is an abundant isoform of NOS in the normal rodent kidney. ${ }^{21,28}$ Marked iNOS mRNA expression has been demonstrated in the S3 segment of the proximal tubules, the cortical and medullary thick ascending limbs and the cortical and inner medullary collecting ducts, whereas minimal to mild iNOS expression was present in the thin limbs of Henle and medullary IC. ${ }^{21,28}$

Although it is well established that NO exhibits vasomodulatory effects and inhibits the development of contractile force in the cardiovascular system, ${ }^{29}$ the exact role that iNOS and its cardiac expression plays in cardiac pathophysiology is poorly understood. It has been reported that $\mathrm{NOS}^{-/-}$mice have a HR similar to that of wild-type mice, as well as normal blood pressure,$^{31}$ and variable and conflicting results exist in the literature regarding cardiac iNOS expression. For example, one study reported that overexpression of iNOS in the heart in mice has no effects on cardiac physiology and that the heart can tolerate high levels of iNOS activity without detrimental functional consequences. ${ }^{31}$ In contrast, another study reported an increase in ventricular size and abnormal conduction in a transgenic mouse model overexpressing iNOS in cardiac myocytes. ${ }^{32}$ In both the normal and hypertensive mice in the present study, there was a high expression of iNOS in cardiac myocytes. Marked iNOS expression has been demonstrated in cardiac myocytes from normal aged (16 months), but not young (6 months), mice. This may suggest that iNOS contributes to age-related ventricular dysfunction. A study by Yang et al. provided evidence for iNOS contribution to age-related ventricular dysfunction. ${ }^{33}$ However, aged iNOS-knockout mice had ventricular function similar to that in younger mice. ${ }^{33}$ In another study, iNOS mRNA and protein levels in the left ventricle were elevated in 18-week-old Dahl salt-sensitive (DS) hypertensive rats, but not in 11-week-old DS rats. ${ }^{34}$ The mice in the present study were younger (4-5 months old) than the mice used in the study of Yang et al. ${ }^{33}$

In the present study, moderate iNOS expression was observed in alveolar macrophages and alveolar septa in normal mice, whereas downregulated (minimal) expression was observed in hypertensive mice. High iNOS expression in the normal lung is consistent with previous studies that showed moderate to strong iNOS expression in alveolar macrophages in normal bovine and rat lungs. ${ }^{35,36}$ There is increasing evidence that free radicals play a role in the pathogenesis of hypertension. ${ }^{37}$ Inducible NOS can produce considerable amounts of free radicals that can react with NO to form peroxynitrite. ${ }^{38}$ The downregulation of iNOS expression in alveolar macrophages and alveolar septa in hypertensive mice in the present study may be related to a unique innate pulmonary defence mechanism of alveolar macrophages to counteract and inhibit the production of free radicals during hypertension.
Nitric oxide plays an important role in various pulmonary epithelial physiological processes. ${ }^{39}$ Some studies have suggested that pulmonary EC are the primary source of expired NO. ${ }^{40,41}$ The role iNOS plays in the lung depends on the cell type in which it is expressed and the localization of that cell type within the lung. The upregulation of iNOS expression in the pulmonary bronchial epithelium in hypertensive mice in the present study suggests that iNOS may play a role in regulating hypoxia induced during hypertension. Spontaneously hypertensive rats not only have systemic hypertension, but also pulmonary arterial hypertension, ${ }^{43}$ and chronic hypoxia-induced pulmonary hypertension is accentuated in this model.$^{43}$ In the present study, both bronchial SMC and pulmonary VSMC showed upregulation of iNOS expression during hypertension compared with controls. This could be related to AngII activity in the transgenic mice used in the present study. Angiotensin II is a potent vasoconstrictor of vascular smooth muscle and has been found to potentiate bronchoconstriction of rabbit airway smooth muscle. ${ }^{44}$

Nitric oxide has been identified as an endothelium-derived relaxing factor in airway smooth muscle and vascular cells. ${ }^{45}$ In addition, NO contributes to oxidative stress. Increased production of free radicals and oxidative stress has been shown to impair endothelium-dependent vasodilatation in hypertension and result in endothelial dysfunction, which usually improves after administration of anti-oxidants. ${ }^{46,47}$ In the present study, there was a significant downregulation of iNOS expression in pulmonary vascular EC in control mice, whereas iNOS expression in pulmonary vascular EC from hypertensive mice was upregulated. These results suggest that EC dysfunction that occurs in hypertension alters iNOS production by vascular EC. It has been found that iNOS impairs NO-dependent vasorelaxation. ${ }^{45}$ The differential responses between airway smooth muscle and vascular cells may be explained by the different levels of NO, different sites of NO production, different actions of NO and different sources of $\mathrm{NO}{ }^{45}$

In conclusion, iNOS regulation in hypertension is complex and depends on the cell type in which it is expressed and the localization of the cell type in the cardiorenal and pulmonary systems. Experiments using iNOS-specific inhibitors are needed to further characterize the contribution of this NOS isoform in hypertension in the cardiorenal and pulmonary systems.

\section{ACKNOWLEDGEMENTS}

The authors thank Dr Curt Sigmund (The University of Iowa Medical School, Iowa City, IA, USA) for providing the transgenic mouse colony and Theresa Cody and Robert Ostroski for technical assistance.

\section{REFERENCES}

1. Brown NJ. Eplerenone: Cardiovascular protection. Circulation 2003; 107: 2512-18.

2. Thompson MW, Smith SB, Sigmund CD. Regulation of human renin mRNA expression and protein release in transgenic mice. Hypertension 1996; 28: 290-296.

3. Lavoie JL, Sigmund CD. Minireview: Overview of the renin-angiotensin system: An endocrine and paracrine system. Endocrinology 2003; 144: 2179-83.

4. Lee MA, Bohm M, Paul M, Bader M, Ganten U, Ganten D. Physiological characterization of the hypertensive transgenic rat TGR (mREN2). Am. J. Physiol 1996; 270: E919-29. 
5. Shimokama T, Haraoka S, Horiguchi H, Sugiyama F, Murakami K, Watanabe T. The Tsukuba hypertensive mouse (transgenic mouse carrying human genes for both renin and angiotensinogen) as a model of human malignant hypertension: Development of lesions and morphometric analysis. Virchows Arch. 1998; 432: 169-75.

6. Pieruzzi F, Abassi ZA, Keiser HR. Expression of renin-angiotensin system components in the heart, kidneys, and lungs of rats with experimental heart failure. Circulation 1995; 92: 3105-12.

7. Fukamizu A, Sugimura K, Takimoto E et al. Chimeric renin-angiotensin system demonstrates sustained increase in blood pressure of transgenic mice carrying both human renin and human angiotensinogen genes. J. Biol. Chem. 1993; 268: 11 617-21.

8. Kai T, Shimada S, Kurooka A, Takenaka T, Ishikawa K. Tissue angiotensin II concentration in the heart and kidneys in transgenic Tsukuba hypertensive mice. Blood Press. 1998; 7: 61-3.

9. Noris M, Remuzzi G. Evidence for a role of nitric oxide in hypertension and in renal disease in man. Contrib. Nephrol. 1996; 119: 8-15.

10. Mattson DL, Maeda CY, Bachman TD, Cowley Jr AW. Inducible nitric oxide synthase and blood pressure. Hypertension 1998; 31: 15-20.

11. Johnson RA, Freeman RH. Renin release in rats during blockade of nitric oxide synthesis. Am. J. Physiol. 1998; 266: 1723-9.

12. Pollock DM, Polakowski JS, Divish BJ, Opgenorth TJ. Angiotensin blockade reverses hypertension during long-term nitric oxide synthase inhibition. Hypertension 1993; 21: 660-6.

13. Merrill DC, Thompson MW, Carney CL et al. Chronic hypertension and altered baroreflex responses in transgenic mice containing the human renin and human angiotensinogen genes. J. Clin. Invest. 2003; 97: $1047-55$.

14. Sigmund CD, Jones CA, Kane CM, Wu C, Lang JA, Gross KW. Regulated tissue- and cell-specific expression of the human renin gene in transgenic mice. Circ. Res. 1992; 70: 1070-9.

15. Major TC, Olszewski B, Rosebury W et al. A nonpeptide, piperidine renin inhibitor provides renal and cardiac protection in doubletransgenic mice expressing human renin and angiotensinogen genes. Cardiovasc. Drugs Ther. 2008: 22; 469-78.

16. Ito S. Nitric oxide in the kidney. Cur. Opin. Nephrol. Hypertens. 1995; 4: $23-30$.

17. Kurtz A, Wagner C. Role of nitric oxide in the control of renin secretion. Am. J. Physiol. 1998; 275: 849-62.

18. Deng AY, Rapp JP. Locus for the inducible, but not a constitutive, nitric oxide synthase cosegregates with blood pressure in the Dahl saltsensitive rat. J. Clin. Invest. 1995; 95: 2170-7.

19. Ihrig M, Dangler CA, Fox JG. Mice lacking inducible nitric oxide synthase develop spontaneous hypercholesterolaemia and aortic atheromas. Atherosclerosis 2001; 156: 103-7.

20. Mohaupt MG, Elzie JL, Ahn KY, Clapp WL, Wilcox CS, Kone BC. Differential expression and induction of mRNAs encoding two inducible nitric oxide synthases in rat kidney. Kidney Int. 1994; 46: 653-65.

21. Ahn KY, Mohaupt MG, Madsen KM, Kone BC. In situ hybridization localization of mRNA encoding inducible nitric oxide synthase in rat kidney. Am. J. Physiol. 1994; 267: 748-57.

22. Morrissey JJ, McCracken R, Kaneto H, Vehaskari M, Montani D, Klahr S. Location of an inducible nitric oxide synthase mRNA in the normal kidney. Kidney Int. 1994; 45: 998-1005.

23. Mattson DL, Higgins DJ. Influence of dietary sodium intake on renal medullary nitric oxide synthase. Hypertension 1996; 27: 688-92.

24. Agmon Y, Peleg H, Greenfeld Z, Rosen S, Brezis M. Nitric oxide and prostanoids protect the renal outer medulla from radiocontrast toxicity in the rat. J. Clin. Invest. 1994; 94: 1069-75.

25. Brezis M, Heyman SN, Dinour D, Epstein FH, Rosen S. Role of nitric oxide in renal medullary oxygenation. Studies in isolated and intact rat kidneys. J. Clin. Invest. 1991; 88: 390-5.

26. Pallone TL, Mattson DL. Role of nitric oxide in regulation of the renal medulla in normal and hypertensive kidneys. Curr. Opin. Nephrol. Hypertens. 2002; 11: 93-8.
27. Chu S, Zhao G, Li D. Effects of captopril on the tissue nitric oxide synthase in offspring from treated pregnant SHRs. Zhonghua Yi Xue Za Zhi 1997; 77: 843-6 (in Chinese with an English abstract).

28. Shin SJ, Lai FJ, Wen JD et al. Increased nitric oxide synthase mRNA expression in the renal medulla of water-deprived rats. Kidney Int. 1999; 56: 2191-202.

29. Kelm M, Schäfer S, Dahmann R et al. Nitric oxide induced contractile dysfunction is related to a reduction in myocardial energy generation. Cardiovasc. Res. 1997; 36: 185-94.

30. Ullrich R, Bloch KD, Ichinose F, Steudel W, Zapol WM. Hypoxic pulmonary blood flow redistribution and arterial oxygenation in endotoxin-challenged NOS2-deficient mice. J. Clin. Invest. 1999; 104: 1421-9.

31. Heger J, Gödecke A, Flögel U et al. Cardiac-specific overexpression of inducible nitric oxide synthase does not result in severe cardiac dysfunction. Circ. Res. 2002; 90: 93-9.

32. Butany J, Stewart DJ, Husain M. Cardiomyocyte overexpression of iNOS in mice results in peroxynitrite generation, heart attack, and sudden death. J. Clin. Invest. 2002; 109: 735-43.

33. Yang B, Larson DF, Watson RR. Modulation of iNOS activity in agerelated cardiac dysfunction. Life Sci. 2004; 75: 655-67.

34. Horinaka S, Kobayashi N, Mori Y, Yagi H, Onoda M, Matsuoka H. Expression of inducible nitric oxide synthase, left ventricular function and remodeling in Dahl salt-sensitive hypertensive rats. Int. J. Cardiol. 2003; 91: 25-35.

35. Liu HW, Anand A, Bloch K, Christiani D, Kradin R. Expression of inducible nitric oxide synthase by macrophages in rat lung. Am. J. Respir. Crit. Care Med. 1997; 156: 223-8.

36. Radi ZA, Caverly J, Dixon RA, Brogden KA, Ackermann MR. Effects of the synthetic selectin inhibitor TBC1269 on tissue damage during acute Mannheimia haemolytica-induced pneumonia in neonatal calves. Am. J. Vet. Res. 2001; 62: 17-22.

37. Wu R, Millette E, Wu L, de Champlain J. Enhanced superoxide anion formation in vascular tissues from spontaneously hypertensive and desoxycorticosterone acetate-salt hypertensive rats. J. Hypertens. 2001; 19: 741-8.

38. Valko M, Leibfritz D, Moncol J, Cronin MT, Mazur M, Telser J. Free radicals and antioxidants in normal physiological functions and human disease. Int. J. Biochem. Cell Biol. 2007; 39: 44-84.

39. Gaston B, Drazen JM, Loscalzo J, Stamler JS. The biology of nitrogen oxides in the airways. Am. J. Respir. Crit. Care Med. 1994; 149: 538-51.

40. Gustafsson LE, Leone AM, Persson MG, Wiklund NP, Moncada S. Endogenous nitric oxide is present in the exhaled air of rabbits, guinea pigs and humans. Biochem. Biophys. Res. Commun. 1991; 181: 852-7.

41. Dweik RA, Laskowski D, Abu-Soud HM et al. Nitric oxide synthesis in the lung. Regulation by oxygen through a kinetic mechanism. J. Clin. Invest. 1998; 101: 660-6.

42. Janssens SP, Thompson BT, Spence CR, Hales CA. Functional and structural changes with hypoxia in pulmonary circulation of spontaneously hypertensive rats. J. Appl. Physiol. 1994; 77: 1101-7.

43. McMurtry IF, Petrun MD, Tucker A, Reeves JT. Pulmonary vascular reactivity in the spontaneously hypertensive rat. Blood Vessels 1979; 16: $61-70$.

44. Yamawaki I, Tamaoki J, Yamauchi F, Konno K. Angiotensin II potentiates neurally mediated contraction of rabbit airway smooth muscle. Respir. Physiol. 1992; 89: 239-47.

45. Gunnett CA, Lund DD, Chu Y, Brooks 2nd RM, Faraci FM, Heistad DD. NO-dependent vasorelaxation is impaired after gene transfer of inducible NO-synthase. Arterioscler. Thromb. Vasc. Biol. 2001; 21: 1281-7.

46. Newaz MA, Nawal NN. Effect of alpha-tocopherol on lipid peroxidation and total antioxidant status in spontaneously hypertensive rats. Am. J. Hypertens. 1998; 11: 1480-5.

47. Park JB, Touyz RM, Chen X, Schiffrin EL. Chronic treatment with a superoxide dismutase mimetic prevents vascular remodeling and progression of hypertension in salt-loaded stroke-prone spontaneously hypertensive rats. Am. J. Hypertens. 2002; 15: 78-84. 